\title{
Development in Rubber Preparation for Endoscopic Training Simulator
}

\author{
D. Surangsrirat, ${ }^{1}$ A. Tongkratoke,${ }^{2}$ S. Samphanyuth, ${ }^{1}$ T. Sununtachaikul, ${ }^{1}$ \\ and A. Pramuanjaroenkij ${ }^{2}$ \\ ${ }^{1}$ Biomedical Signal Processing Laboratory, National Electronics and Computer Technology Center, \\ 112 Thailand Science Park, Phahonyothin Road, Khlong Nueng, Khlong Luang, Pathum Thani 12120, Thailand \\ ${ }^{2}$ Department of Mechanical and Manufacturing Engineering, Faculty of Science and Engineering, \\ Kasetsart University, Chalermphrakiat Sakon Nakhon Province Campus, Sakon Nakhon 47000, Thailand
}

Correspondence should be addressed to A. Pramuanjaroenkij; anchasa@gmail.com

Received 18 February 2016; Revised 14 June 2016; Accepted 15 June 2016

Academic Editor: Somchai Thongtem

Copyright (C) 2016 D. Surangsrirat et al. This is an open access article distributed under the Creative Commons Attribution License, which permits unrestricted use, distribution, and reproduction in any medium, provided the original work is properly cited.

\begin{abstract}
Endoscopy is one of the most important procedures in diagnosis and treatment of gastrointestinal tract problems. While endoscopic procedure has tremendous benefits, physicians require considerable practice and time to develop competency. Current endoscopic training process involves cognitive learning and hands-on training under the supervision of an expert gastroenterologist. Previous studies have shown that fellow involvement prolongs procedural time and incurs additional expenses to the institution. Moreover, the patient also experiences more discomfort and injury risk. Introduction of training simulator into the training process could reduce the involvement of the patients and thus reduce the risk. Porcine model is commonly used for training in endoscopy due to the similar tactile response to a human gastrointestinal tract. However, information on elastic behavior of pig or human gastrointestinal tract for the engineering purposes was limited. In this study, the modulus of elasticity and ultimate tensile stress data of the pig stomach and intestines, small and large intestines, were measured and compared with multiple rubber stomach and intestines models. Based on the experimental results and experienced gastroenterologists feedback, the proposed dipped rubber composition can provide a satisfactory tactile feedback and could be used to simulate a human gastrointestinal tract for an endoscopic simulation training model.
\end{abstract}

\section{Introduction}

Endoscopy is a procedure for examining a gastrointestinal (GI) tract. Different type of endoscope, usually a long flexible tube with a light and camera attached to the tip, is used depending on the procedure. For example, upper endoscopy uses gastroscope for examination of the lining of the upper part of the GI tract: esophagus, stomach, and duodenum. Colonoscopy uses colonoscope for examination of the lining of the large intestine: rectum and colon. These procedures are also called minimally invasive surgery since the diagnosis and treatments can be done with reduced invasion. The statistical data from World Cancer Report by World Health Organization (WHO) shows that gastrointestinal tract cancers are among the leading causes of death from cancer [1]. The treatment, especially at the earlier stage, can be done and will reduce death rates significantly $[2,3]$. While endoscopic procedure has tremendous benefits, physicians require considerable practice and time to develop competency. Current endoscopic training process involves cognitive learning and hands-on training under the supervision of an expert gastroenterologist. Previous studies showed that fellow involvement prolongs procedural time and incurs additional expenses to the institution [4]. Moreover, the patient also experiences more discomfort and injury risk. Introduction of training simulator into the training process could reduce the involvement of the patients and thus reduce the risk [5]. In a review of adverse events, Panteris et al. reviewed the perforation which was the result of endoscopic manipulation and found the frequency of perforation to be 1 in 1400 for overall colonoscopies and 1 in 1000 for therapeutic colonoscopies [6]. 


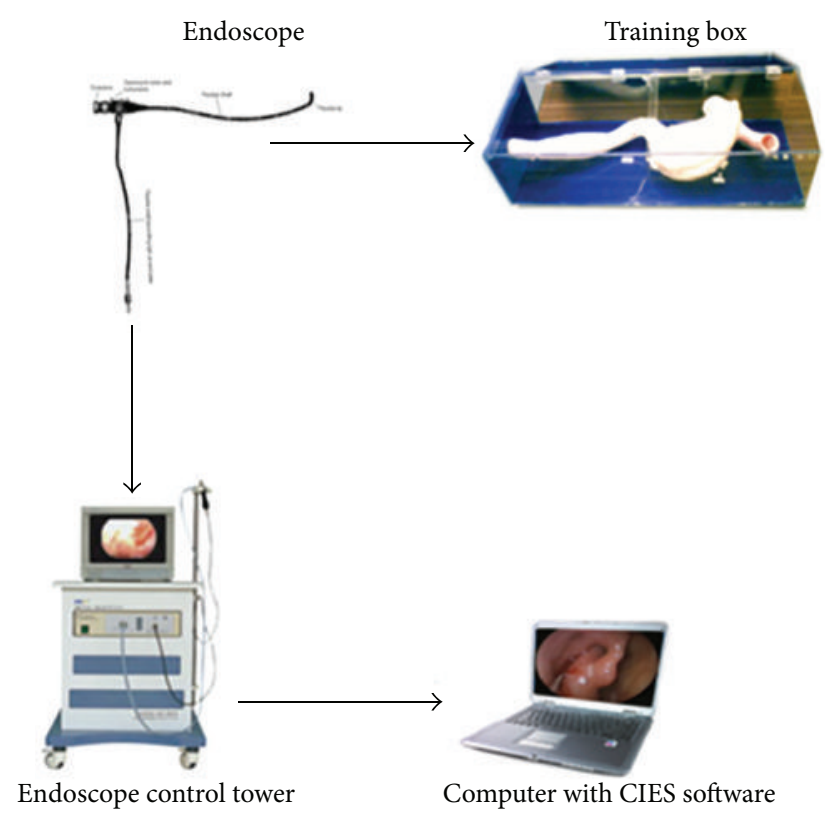

FIGURE 1: A low-cost training simulator proposed by Surangsrirat et al. [7].

American Society for Gastrointestinal Endoscopy (ASGE) published information for principles of training in gastrointestinal endoscopy and emphasized that the training should follow a natural progression [8]. They also recommend that gastroenterology fellows should perform a minimum of 130 upper endoscopy procedures while the joint advisory group for endoscopy in Britain recommends the minimum of 300 procedures and more for colonoscopy to become competent [9]. The current training method relies heavily on performing an endoscopy on patient under the supervision of an experienced gastroenterologist. Recently, the use of simulators becomes a viable alternative for endoscopic training. Fellows can experience the manual manipulations of an endoscope without sacrificing patient comfort and safety. There are several types of training simulator for endoscopic training. Porcine model is commonly used for upper endoscopy training. Virtual reality computer simulator allows training for both upper and lower endoscopy. However, substantial investment is required in order to setup the training facility for both animal model and virtual reality simulator.

In the previous study, Surangsrirat et al. proposed a computer integrated endoscopic simulator for training in upper endoscopy as a low-cost alternative to the traditional training methods and virtual reality simulators as shown in Figure 1 [7]. Fellow could train basic maneuvering skills of the endoscope to achieve certain competency before training on patient. For endoscopic training simulator, a human GI tract can be fabricated by using resin or natural rubber (Hevea brasiliensis), so called Para rubber. In comparison with plastic and resin, mold for rubber fabrication is easier and cheaper to create. Para rubber is chosen as a raw material to simulate the GI tract because of its elastomeric chemical property which makes this material elastic and durable. The rubber appearance can also be made to closely resemble a human GI tract.

Further investigations on using rubber to simulate human GI tract were conducted. Surangsrirat et al. introduced their first experimental results of rubber samples which were prepared by rubber casting technique [10]. Since there was a limited number of usable elastic behavior information on both pig and human stomachs, the modulus of elasticity and ultimate tensile stress data of the pig stomach samples were evaluated and compared with those data of the casted rubber samples. They found that the casted rubber with normal 668/60 formula and soft surface revealed the closest average modulus of elasticity and the closest average ultimate tensile stress to those of the pig samples, in comparison with other casted rubber samples. Pramuanjaroenkij et al. presented the experimental evaluations of the stomach rubber samples, which were prepared by rubber dipping technique, and compared properties of the dipped rubber samples with the casted rubber samples [11]. The pig stomach was tested for the elastic behavior properties: the modulus of elasticity and ultimate tensile stress data. Then, different rubber samples from the casting and dipping techniques were prepared and tested by a universal testing machine for tensile test. Based on their experiments, the casted rubber with $668 / 60$ formula and 2-minute dipped rubber samples showed the closest average modulus of elasticity and the closest average ultimate tensile stress to those of the pig stomach samples.

The objective of this research is to experimentally investigate the elastic properties of pig stomach, pig intestine, and rubber samples from the casting and dipping techniques to find a suitable rubber composition and fabrication technique that could represent a human GI tract for an endoscopic training simulator. Samples with the most suitable modulus of elasticity and ultimate tensile stress combining with the assessment of tactile feedback from experienced gastroenterologists will be used to fabricate human GI tract for a lowcost endoscopic training simulator. Lastly, the simulator will be tested by experienced gastroenterologists and fellows in the operating room with actual operating room equipment and setup to validate the usefulness of the simulator in the fellow training process.

\section{Methods}

For our experiment, the goal is to find a rubber composition that could replicate a human gastrointestinal (GI) tract and provide similar tactile feedback. Therefore, rubber samples with multiple compositions preparing by the dipping and casting techniques are fabricated into the shape of human stomachs and intestines. Fresh pig stomachs and intestines are also prepared for the experiment. In order to quantitatively select a suitable rubber composition with similar elastic properties to the pig organs, the modulus of elasticity and ultimate tensile stress are chosen as indicators. Based on Hooke's law, the elastic stress is linearly related to elastic strain by means of the modulus of elasticity as

$$
S=E \varepsilon,
$$




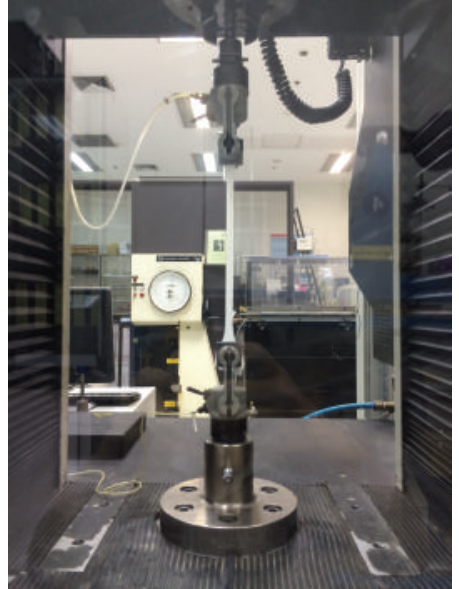

FIGURE 2: Tensile test using universal testing machine 8872, Instron 8872 Servohydraulic UTM.

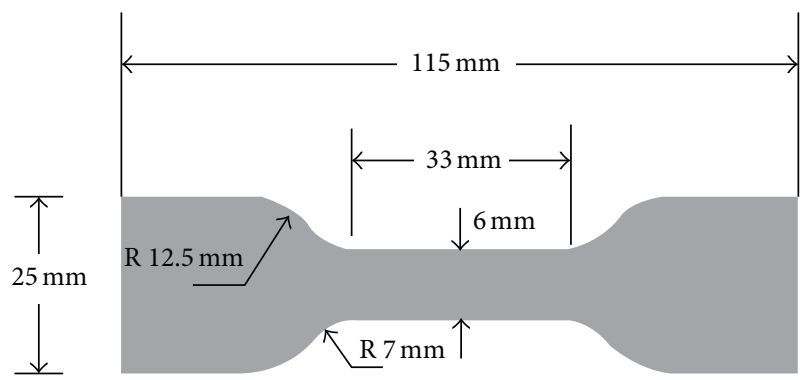

FIgURE 3: The dimension of dumbbell shaped specimen for the tensile test.

where $S$ is elastic stress, $E$ is modulus of elasticity or Young's modulus, and $\varepsilon$ is elastic strain [12]. The basic data on the mechanical properties of a material can be obtained from the tension test. Load and elongation are measured at frequent intervals during the test and expressed as average stress and strain according to Hooke's law. The greatest stress that a sample can withstand without experiencing a permanent strain when the load is removed is defined as an elastic limit. The slope of a stress-strain curve in this region is a modulus of elasticity. However, the elastic limit is tedious; therefore the proportional limit is often replaced and this limit is the stress at which the stress-strain curve deviates from linearity. For engineering purposes, the limit of usable elastic behavior is described by the yield strength. Yield strength is defined as the stress which will produce a small amount of permanent deformation. Eventually, the load reaches a maximum value and the maximum load divided by the original area of the specimen is the ultimate tensile strength.

Figure 2 shows a universal testing machine 8872, Instron 8872 Servohydraulic UTM, which was used for tensile test in our experiments. The tensile test followed ASTM D412 standard test method for rubber and elastomers. The samples were cut into equal size dumbbell shaped specimen with the dimension as illustrated in Figure 3. Each specimen was loaded into the tensile grips. The test began by separating tensile grips at the speed of 500 millimeter per minute. The grips stopped after the sample break. For the first experiment,
TABLE 1: The composition of the main casted rubber sample used in the experiment.

\begin{tabular}{lc}
\hline Ingredient & Amount (gram) \\
\hline $60 \%$ latex & 668 \\
10\% potassium oleate & 60 \\
$50 \%$ sulfur & 16 \\
$50 \%$ ZDEC & 8 \\
$50 \%$ ZMBT & 8 \\
$50 \%$ Wingstay L & 8 \\
$50 \%$ zinc oxide & 40 \\
$33 \%$ DPG & 8 \\
\hline
\end{tabular}

TABLE 2: The composition of the main dipped rubber sample used in the experiment.

\begin{tabular}{lc}
\hline Ingredient & Amount (gram) \\
\hline $60 \%$ latex & 100 \\
10\% potassium hydroxide & 0.25 \\
10\% potassium laurate & 0.2 \\
$50 \%$ sulfur & 1.25 \\
$50 \%$ ZDEC & 0.4 \\
$50 \%$ ZMBT & 1 \\
$50 \%$ Wingstay L & 1 \\
$50 \%$ zinc oxide & 1 \\
$33 \%$ DPG & 1.5 \\
$50 \%$ calcium carbonate & 22.5 \\
\hline
\end{tabular}

a relationship between the rubber and the pig stomach samples was investigated. Figures 4 and 5 show the chosen pig stomachs and stomach shaped rubber samples, respectively. Two main rubber fabrication techniques, the casting and dipping, were used to fabricate stomach shaped rubber samples. The composition of the main casting and dipping rubber samples is listed in Tables 1 and 2, respectively. Both pig stomachs and rubber samples were cut into dumbbell shaped specimen for the tensile test. The total of 10 pig stomach specimens was used in this experiment. For the rubber samples, 5 specimens for each of the 7 different rubber compositions, 3 for casted rubber, and 4 for dipped rubber, for the total of 35 rubber specimens, were prepared. The modulus of elasticity and ultimate tensile stress of each specimen were measured and recorded. Then, the properties measured from the stomach and rubber specimens were compared and evaluated to find the closest elastic behavior between the two. The rubber samples with closest properties will be selected and evaluated for their tactile feedback by experienced gastroenterologists.

In the second experiment, we would like to verify that a relationship between the rubber and pig intestine samples was similar to the first experiment. Since the small and large intestines have different characteristics, the pig intestine was separated into the small intestine and the large intestine as shown in Figure 6. Based on the evaluation from experienced gastroenterologists in the previous experiment, the casted stomach shaped rubber samples could not provide satisfactory tactile feedback, even though some of the qualitative 

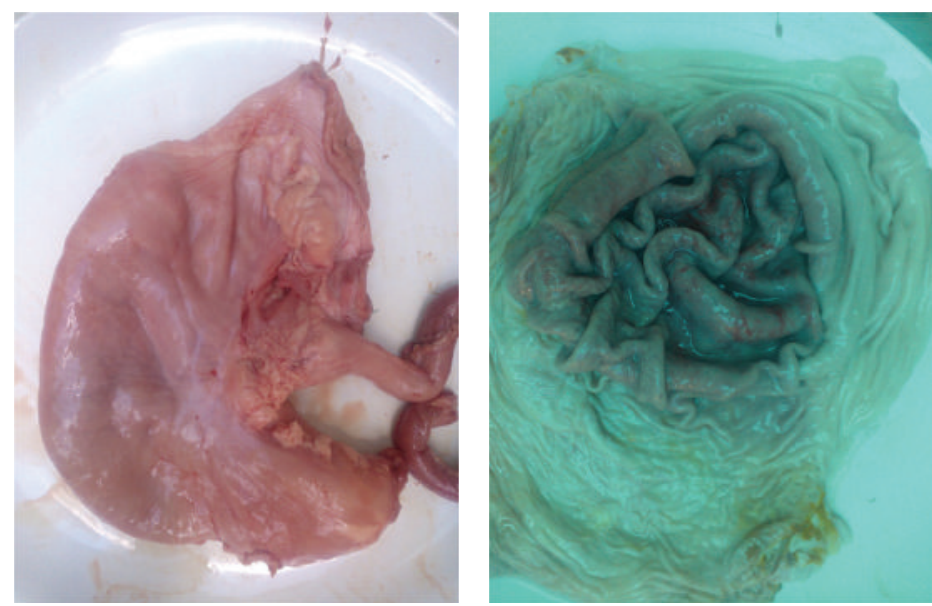

FIGURE 4: Pig stomach samples before the tensile test preparation process.
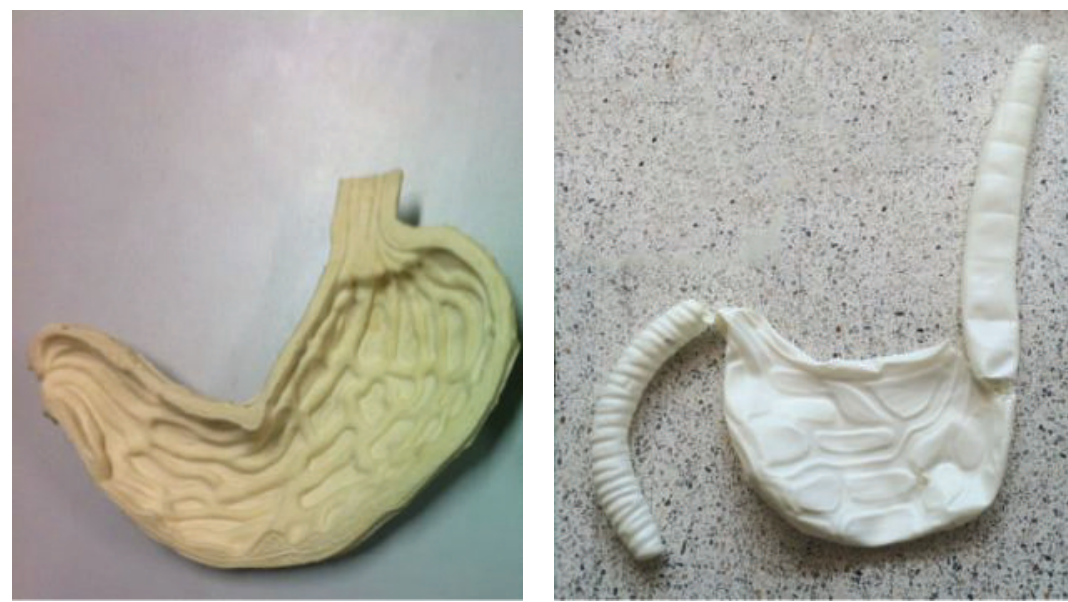

FIgURE 5: Casted and dipped stomach shaped rubber samples.
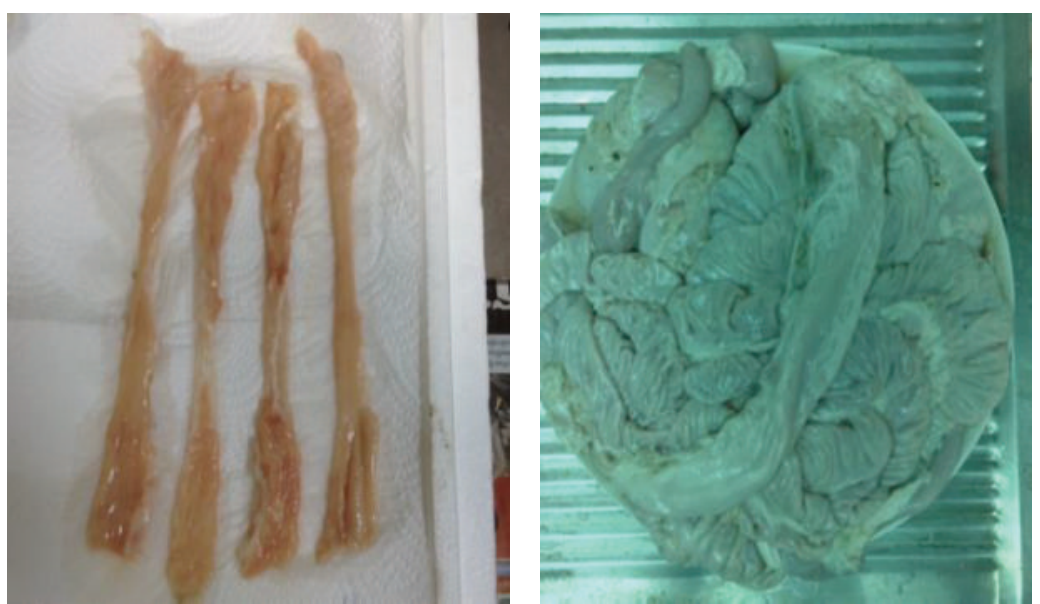

FIGURE 6: Pig small and large intestines samples. 

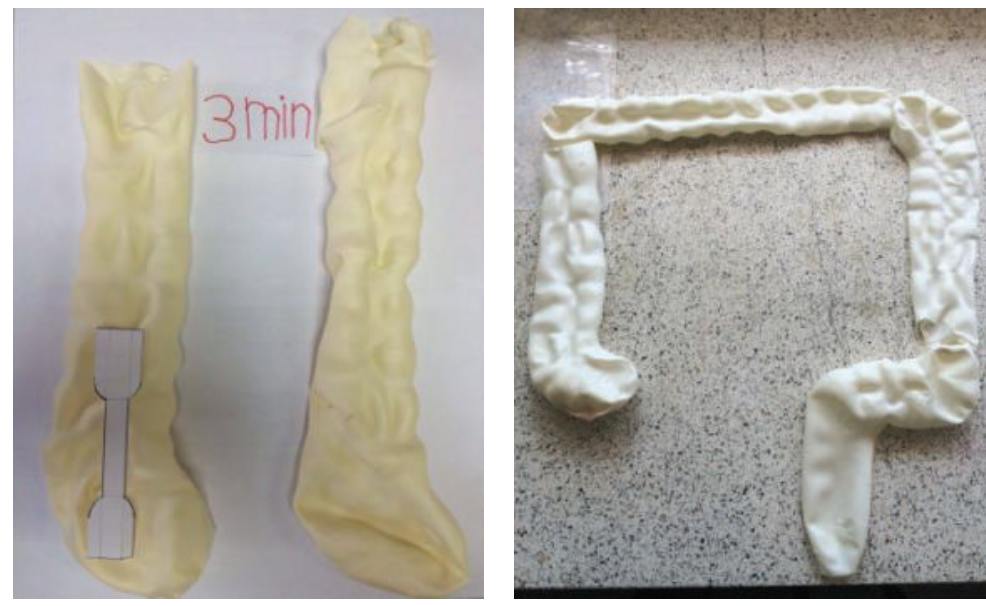

FIGURE 7: Dipped intestine shaped rubber samples.

TABLE 3: Average modulus of elasticity and average ultimate tensile stress for the pig stomach samples and casted and dipped rubber samples.

\begin{tabular}{lcc}
\hline Samples & Average modulus of elasticity $(E) ; \mathrm{MPa}$ & Average ultimate tensile stress (UTS); MPa \\
\hline Pig stomach & 0.004567 & 0.24 \\
$668 / 60$ casted & 0.0015 & 0.15 \\
$600 / 70$ casted 1st & 0.0020 & 0.34 \\
$600 / 70$ casted 2nd & 0.0015 & 0.32 \\
3 min dipped & 0.003466 & 2.239 \\
2 min dipped & 0.003566 & 2.644 \\
1 min dipped & 0.003817 & 2.956 \\
20 sec dipped & 0.003053 & 0.240 \\
\hline
\end{tabular}

properties of the rubber were close to the pig stomach. Therefore, only the dipped intestine shaped rubber samples were used in this experiment. Figure 7 shows dipped intestine shaped rubber samples. The total of 10 dumbbell shaped pig intestine specimens was used in this experiment, 5 specimens for small intestine and 5 specimens for large intestine, respectively. For the rubber samples, 3 different dipped rubber compositions for the total of 30 dumbbell shaped rubber specimens were prepared, 15 for small intestine and 15 for large intestine. The modulus of elasticity and ultimate tensile stress of each specimen were measured and recorded for further evaluation.

\section{Results and Discussion}

For the evaluation of the rubber and pig stomach samples, the total of 10 dumbbell shaped pig stomach specimens was used in this experiment. Seven different rubber compositions, 3 for casted and 4 for dipped rubber samples, for the total of 35 dumbbell shaped rubber specimens were prepared, 5 specimens for each composition. The difference between the casted rubber compositions is mainly the ratio of latex and potassium oleate where the difference of the dipped rubber compositions is the dipped duration. Table 3 shows average modulus of elasticity and average ultimate tensile stress for the pig stomach, 3 different compositions of the casted stomach shaped rubber samples and 4 different compositions of the dipped stomach shaped rubber samples. Stress-strain curves for the pig stomach and the casted rubber samples are shown in Figure 8. All of the casted compositions have lower average modulus of elasticity comparing to the pig stomach. However, the $668 / 60$ casted composition has closer average ultimate tensile stress. Therefore, the $668 / 60$ composition was selected as a representation of the casted rubber. Figure 9 shows the stress-strain curves for the pig stomach and the dipped rubber samples. The $1 \mathrm{~min}, 2 \mathrm{~min}$, and $3 \mathrm{~min}$ dipped samples have relatively close average modulus of elasticity comparing to the pig stomach. Both $2 \mathrm{~min}$ and $3 \mathrm{~min}$ dipped samples have a close average ultimate tensile stress. We ultimately selected $2 \mathrm{~min}$ as a representation of the dipped rubber since it has a closer average of modulus of elasticity to the pig stomach.

A close-up view of the stress-strain curves of the representation of the casted and dipped rubber and pig stomach is shown in Figure 10. Despite the fact that the casted rubber sample has similar characteristic to the pig stomach at low tension, the dipped rubber sample characteristic resembles the pig stomach at higher tension. The higher elastic modulus of the dipped rubber sample also implied that the flexibility of dipped sample was high. From the average ultimate tensile stress point of view, the casted rubber sample was closer to the pig sample. The ultimate tensile stress of the dipped rubber sample was significantly greater than both the casted rubber and pig stomach. Nonetheless, higher ultimate tensile stress also implied that the dipped rubber stomach could take higher impact from the gastroenterologist trainees. 


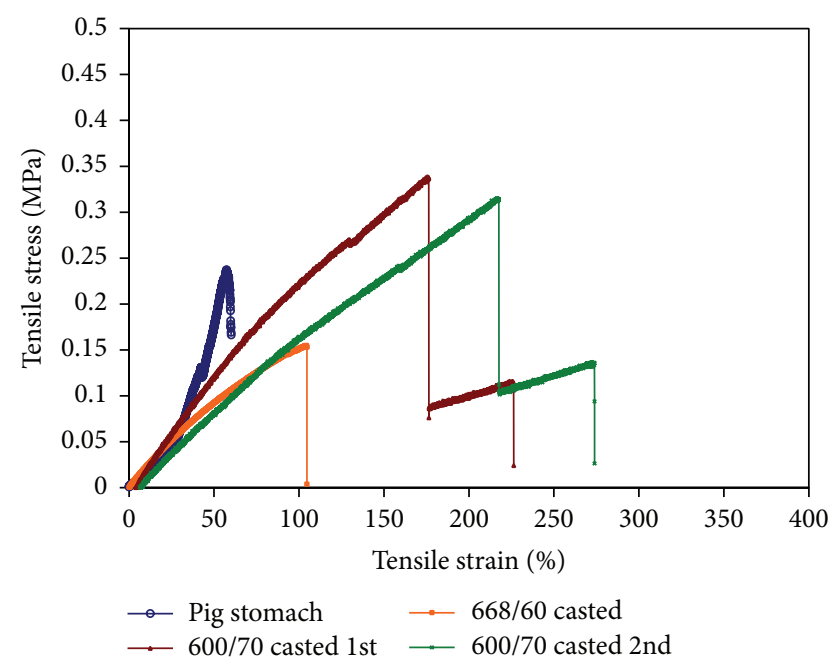

Figure 8: Stress-strain curves for the pig stomach samples and the casted rubber samples.

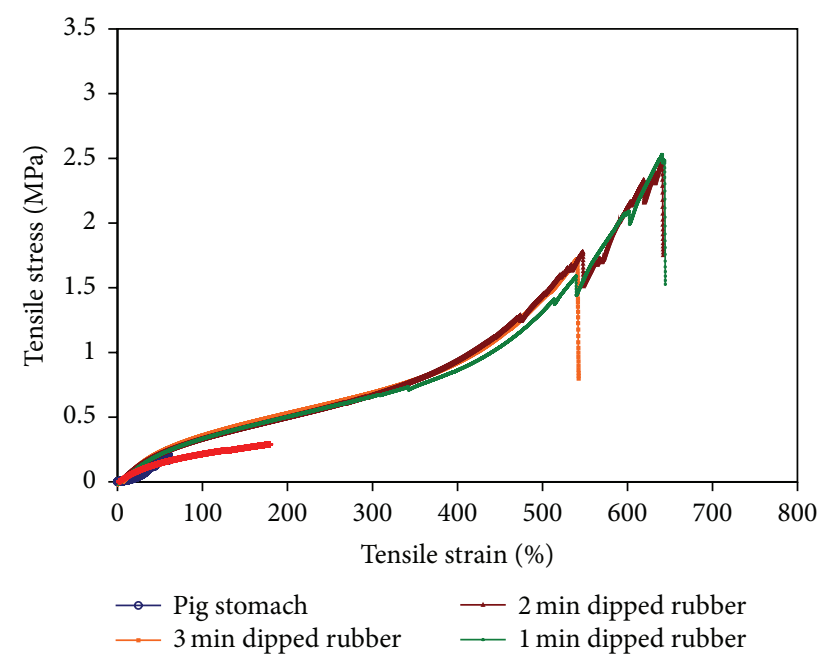

FIGURE 9: Stress-strain curves for the pig stomach samples and the dipped rubber samples.

Therefore, their lifetime could be longer than the casted rubber and would be suitable for multiple training sessions.

Both 668/60 casted and 2 min dipped rubber compositions were used to fabricate the human stomach shaped rubber models in order to validate their tactile feedback by experienced gastroenterologists. Both models were mounted into the endoscopic training simulator ready for the gastroenterologists to perform an upper endoscopy diagnosis. After the performance, gastroenterologists gave similar comments that the tactile feedback of the casted rubber stomach was not realistic and definitely worse than the dipped rubber stomach. They also stated that the tactile feedback of the dipped rubber stomach was acceptable and closely resemble the human stomach. The explanation could be that the dipped rubber has higher modulus of elasticity and the closer characteristic to pig stomach at high tension.

According to the feedback from experienced gastroenterologists in the stomach experiment, only the dipped

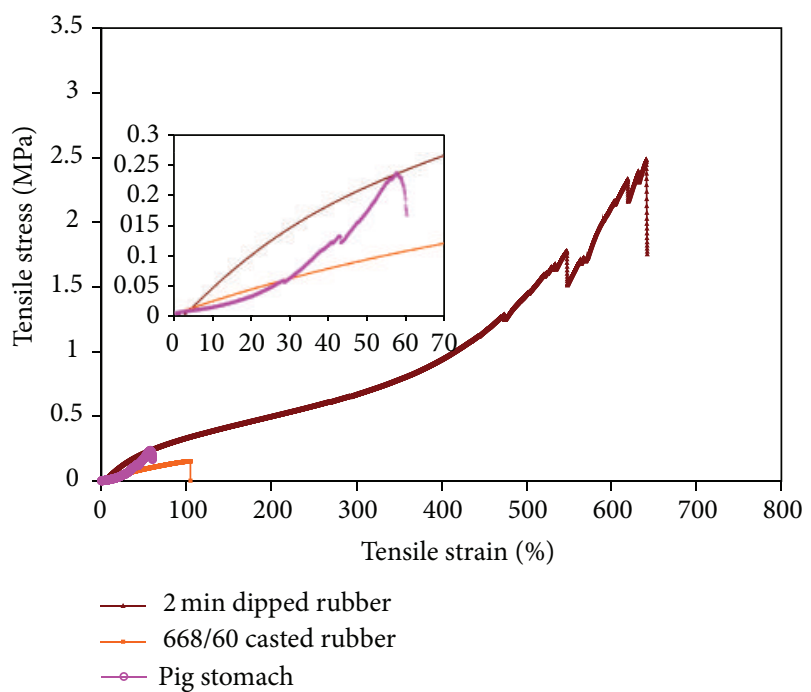

Figure 10: Close-up view of the stress-strain curves for the pig stomach samples and representation of casted and dipped rubber samples.

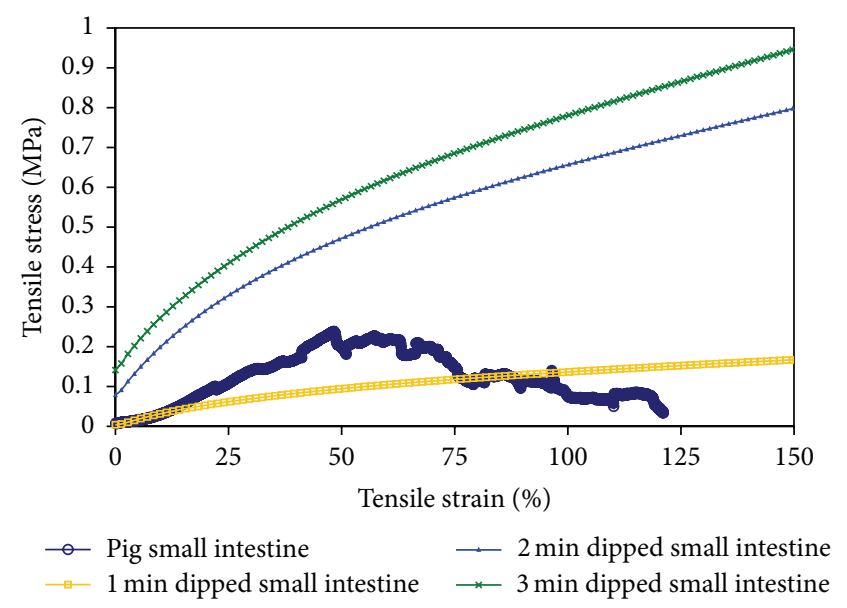

FIGURE 11: Close-up view of the stress-strain curves for the pig small intestine samples and the dipped rubber samples.

intestine shaped rubber samples were used for the intestine experiment. Since the small and large intestines have different characteristics, the pig intestine samples were separated into the small and large intestine. The total of 10 pig intestine specimens was used in this experiment, 5 specimens for small and 5 specimens for large intestines, respectively. For the rubber samples, 3 different dipped rubber compositions for the total of 30 rubber specimens were prepared, 15 specimens for small intestine and 15 specimens for large intestine. Tables 4 and 5 show average modulus of elasticity and average ultimate tensile stress for the dipped intestine shaped rubber samples and the pig small intestine and large intestine, consequently. Since the ultimate tensile stress of the dipped rubber was much higher than the pig intestine, only close-up view of the stress-strain curves for the dipped rubber samples and the pig small and large intestine samples are shown in Figures 11 and 12. Moreover, the biological tissue could be defined as viscoelastic material [13]; the stress-strain 
TABLE 4: Average modulus of elasticity and average ultimate tensile stress for the pig small intestine samples and dipped rubber samples.

\begin{tabular}{lcc}
\hline Samples & Average modulus of elasticity $(E) ; \mathrm{MPa}$ & Average ultimate tensile stress (UTS); MPa \\
\hline Pig small intestine & 0.006161969 & 0.221 \\
3 min dipped & 0.000922296 & 1.417 \\
2 min dipped & 0.002700755 & 9.354 \\
1 min dipped & 0.004656689 & 6.781 \\
\hline
\end{tabular}

TABLE 5: Average modulus of elasticity and average ultimate tensile stress for the pig large intestine samples and dipped rubber samples.

\begin{tabular}{lcc}
\hline Samples & Average modulus of elasticity $(E) ; \mathrm{MPa}$ & Average ultimate tensile stress $(\mathrm{UTS}) ; \mathrm{MPa}$ \\
\hline Pig large intestine & 0.017326077 & 0.514 \\
3 min dipped & 0.002305288 & 4.601 \\
2 min dipped & 0.003308379 & 6.131 \\
l min dipped & 0.003881354 & 4.327 \\
\hline
\end{tabular}

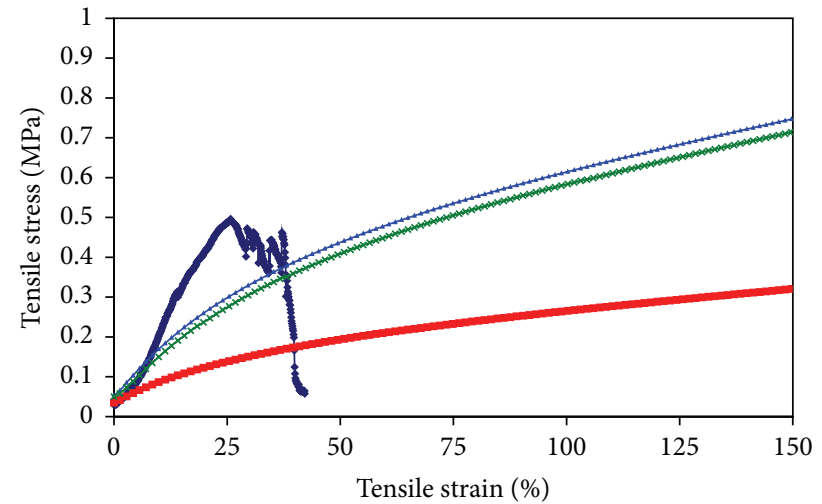

$\longrightarrow$ Pig large intestine $\quad \longrightarrow 2$ min dipped large intestine

$\rightarrow 1$ min dipped large intestine $\quad \ldots 3$ min dipped large intestine

Figure 12: Close-up view of the stress-strain curves for the pig large intestine samples and the dipped rubber samples.

relationship can change as the loading speed or strain rate changes. Since the natural rubber behavior could be defined as elastic material, the viscoelastic and elastic materials could not behave the same but one should take only the interest tension range into account, for example, from $0 \%$ to $150 \%$ tensile strain.

For the small intestine, among the 3 rubber samples, the result clearly shows that 1 min dipped intestine shaped rubber sample has the closest average modulus of elasticity and similar stress-strain curve to the pig small intestine sample. However, for the large intestine, the $2 \mathrm{~min}$ and $3 \mathrm{~min}$ dipped intestine shaped rubber samples have closer stressstrain curve than 1 min sample. The 2 min dipped sample was selected as a representative for the large intestine due to the closer modulus of elasticity. Similar to the stomach result, the ultimate tensile stress of the dipped rubber samples was significantly greater than the pig intestine sample for both small and large intestine. The results also indicate that the properties of small and large intestines are different. Pig large intestine has higher modulus of elasticity and ultimate tensile stress than small intestine. For the lower part of the endoscopic training simulator, colonoscopy is an examination of the lining of the large intestine. We selected 2 min and 3 min dipped rubber sample for the fabrication of a human lower GI tract model to be tested by experienced gastroenterologists. They could not differentiate the $2 \mathrm{~min}$ and 3 min dipped rubber models. However, they feel that the tactile feedback from both models was realistic.

\section{Conclusion}

The modulus of elasticity and ultimate tensile stress data of the pig organs were measured and compared with multiple organ shaped rubber samples. The experimental results for the pig stomach show that casting is not a suitable technique for fabrication of a gastrointestinal (GI) tract for endoscopic training simulator. The dipped stomach shaped rubber has a close modulus of elasticity and similar characteristic to the pig stomach, based on stress-strain curve at high tension. Moreover, it could provide satisfactory tactile feedback to experienced gastroenterologists. Similarly, the experiment results for the pig intestine reveal that the dipped intestine shaped rubber has similar characteristic to the pig intestine, especially the 2 min dipped composition. Therefore, in order for a GI tract rubber model to provide a decent tactile feedback, the modulus of elasticity and characteristic of the stress-strain curve at high tension are important factors. High ultimate tensile stress means that the rubber GI model could sustain high punched stresses from trainees before being damaged. Therefore, a durable endoscopic training simulator could be made and the rubber GI model would be suitable for multiple training sessions.

After the experiment, the proposed rubber composition and fabrication technique was used to make a human GI tract rubber model for a low-cost endoscopic training simulator as shown in Figure 13. The simulator was evaluated by 2 hospitals in Thailand, Thammasat University and Kokha Hospitals, as shown in Figure 14. The evaluation scores were based on (1) face validity which is a combination of visual, structural, tactile feedback, practical use, and overall virtue and (2) content validity which is a practical performing and responding processes and satisfaction consisting of experience augmentation, application promptness, and training 


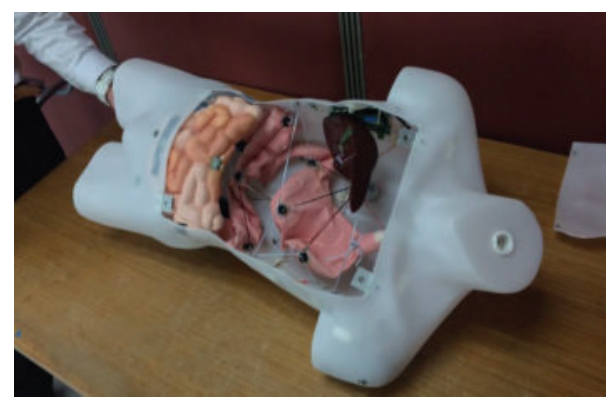

FIgURE 13: A low-cost endoscopic training simulator using a proposed rubber composition and fabrication technique for the human GI tract rubber model.

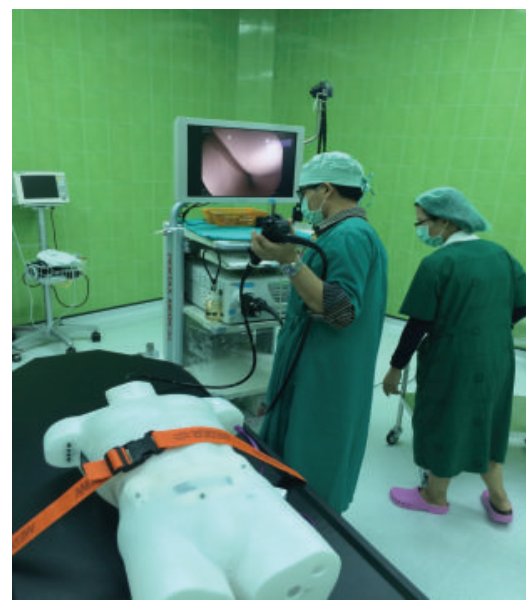

FIGURE 14: A low-cost endoscopic training simulator in the operating room of Kokha Hospital, Thailand.

advantages [14-16]. From total of 10 points, the average face validity and content validity were 7.76 and 7.5 , respectively. Therefore, a low-cost endoscopic training simulator with the proposed rubber composition and fabrication technique could be considered a success and could be used to benefit the fellow training process.

\section{Competing Interests}

The authors declare that there are no competing interests regarding the publishing of this paper.

\section{Acknowledgments}

This work was financially supported by The Thailand Research Fund (TRF). The authors would like to thank everyone at Bualuang Endo-Laparoscopic Telesurgery Center (BELTEC), Thammasat University Hospital, Pathum Thani Province, and Kokha Hospital, Lampang Province, Thailand, for their hospitality and cooperation. They would also like to thank all the participants in this experiment for their cooperation.

\section{References}

[1] B. W. Stewart and C. P. Wild, World Cancer Report, World Health Organization, Geneva, Switzerland, 2014.

[2] A. D. Müller and A. Sonnenberg, "Prevention of colorectal cancer by flexible endoscopy and polypectomy: a case-control study of 32702 veterans," Annals of Internal Medicine, vol. 123, no. 12, pp. 904-910, 1995.

[3] O. Hosokawa, T. Miyanaga, Y. Kaizaki et al., "Decreased death from gastric cancer by endoscopic screening: association with a population-based cancer registry," Scandinavian Journal of Gastroenterology, vol. 43, no. 9, pp. 1112-1115, 2008.

[4] L. B. Gerson and J. Van Dam, "Technology review: the use of simulators for training in GI endoscopy," Gastrointestinal Endoscopy, vol. 60, no. 6, pp. 992-1001, 2004.

[5] R. E. Sedlack, J. C. Kolars, and J. A. Alexander, "Computer simulation training enhances patient comfort during endoscopy," Clinical Gastroenterology and Hepatology, vol. 2, no. 4, pp. 348352, 2004.

[6] V. Panteris, J. Haringsma, and E. J. Kuipers, "Colonoscopy perforation rate, mechanisms and outcome: from diagnostic to therapeutic colonoscopy," Endoscopy, vol. 41, no. 11, pp. 941-951, 2009.

[7] D. Surangsrirat, A. R. Deshpande, S. Surangsrirat, M. A. Tapia, and W. Zhao, "A customized simulation system with computer integrated auto-evaluation function for upper endoscopy training," Technology and Health Care, vol. 19, no. 2, pp. 79-90, 2011.

[8] D. G. Adler, G. Bakis, W. J. Coyle et al., "Principles of training in GI endoscopy," Gastrointestinal Endoscopy, vol. 75, no. 2, pp. 231-235, 2012.

[9] S. Weaver, S. Mohanty, D. Fine, P. Winwood, K. Isaacs, and I. Grimm, "Comparison of endoscopic experience obtained in an American and a British Gastroenterology Training Program," Gastrointestinal Endoscopy, vol. 59, no. 5, article P125, 2004.

[10] D. Surangsrirat, A. Tongkratoke, and A. Pramuanjaroenkij, "A rubber investigations for a gastroscopy training kit," in Proceedings of the The 5th TSME International Conference on Mechanical Engineering, 2014.

[11] A. Pramuanjaroenkij, A. Tongkratoke, and D. Surangsrirat, "Development in rubber preparation for a gastroscopy kit," in Proceedings of the 6th TSME International Conference on Mechanical Engineering, 2015.

[12] G. E. Dieter, Mechanical Metallurgy, McGraw-Hill, Singapore, 1998.

[13] G. Zhang, "Evaluating the viscoelastic properties of biological tissues in a new way," Journal of Musculoskeletal Neuronal Interactions, vol. 5, no. 1, pp. 85-90, 2005.

[14] A. M. Plooy, A. Hill, M. S. Horswill et al., "Construct validation of a physical model colonoscopy simulator," Gastrointestinal Endoscopy, vol. 76, no. 1, pp. 144-150, 2012.

[15] J.-S. Chen, H.-H. Hsu, I.-R. Lai et al., "Validation of a computerbased bronchoscopy simulator developed in Taiwan," Journal of the Formosan Medical Association, vol. 105, no. 7, pp. 569-576, 2006.

[16] F. J. Carter, M. P. Schijven, R. Aggarwal et al., "Consensus guidelines for validation of virtual reality surgical simulators," Surgical Endoscopy and Other Interventional Techniques, vol. 19, no. 12, pp. 1523-1532, 2005. 

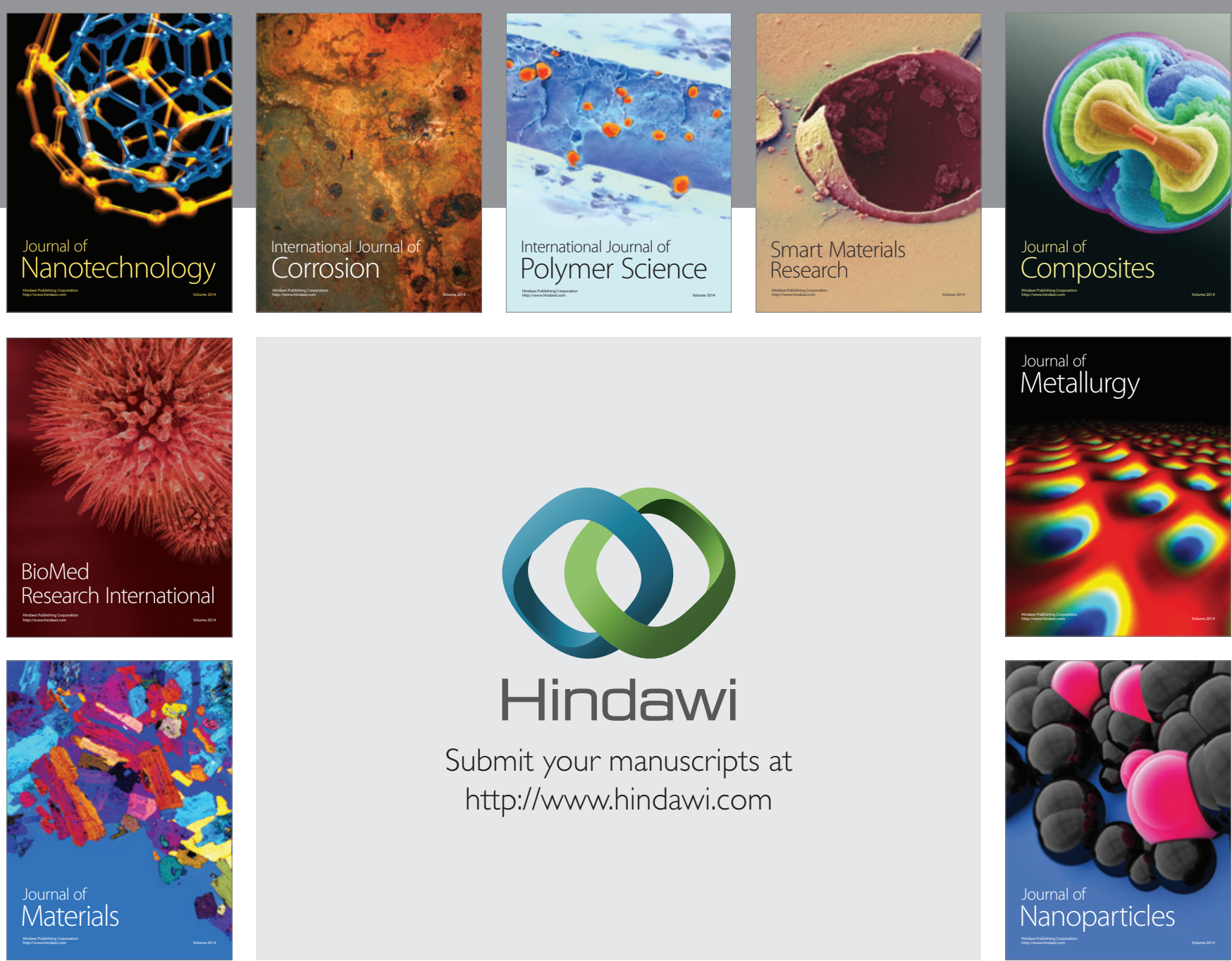

\section{Hindawi}

Submit your manuscripts at

http://www.hindawi.com

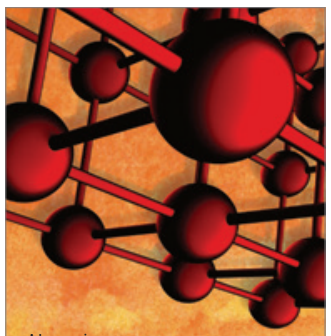

Materials Science and Engineering
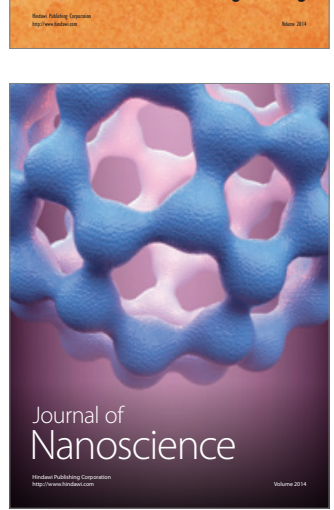
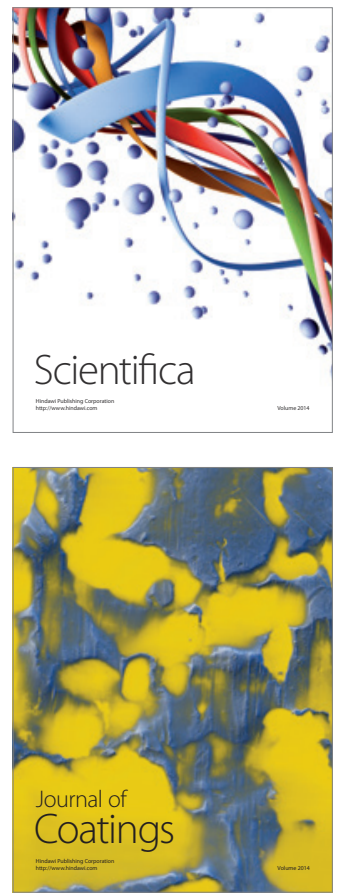
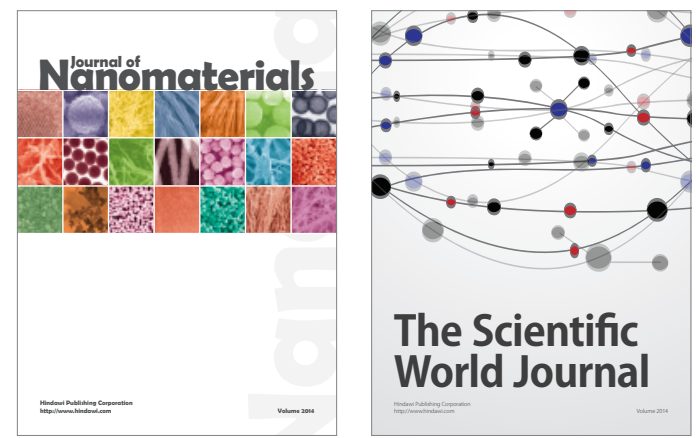

The Scientific World Journal
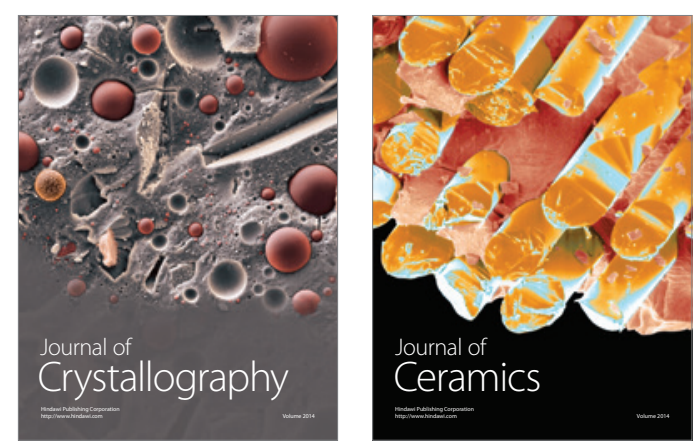
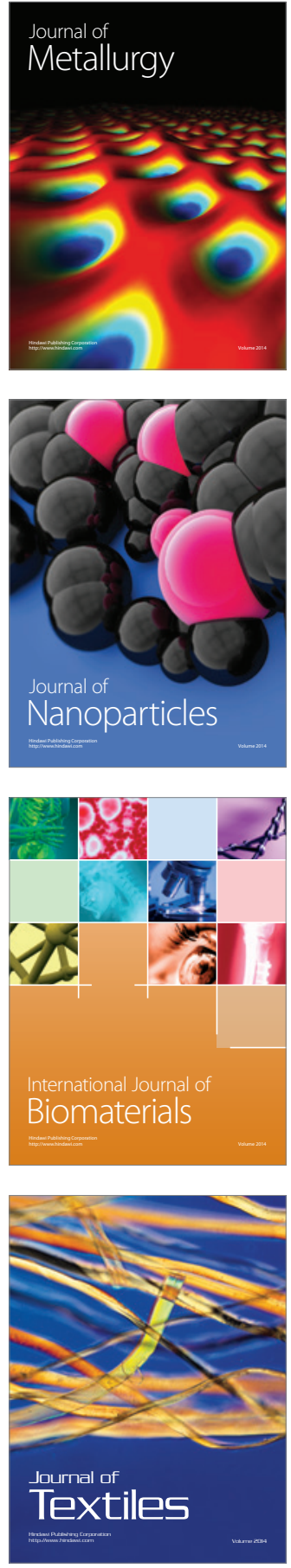